\title{
Presession Posterior Alpha Enhancement May Accelerate Neurofeedback Learning and Response
}

\author{
Revital Yonah \\ Private practice, Jerusalem, Israel \\ BetterFly Neurofeedback, Tel-Aviv, Israel
}

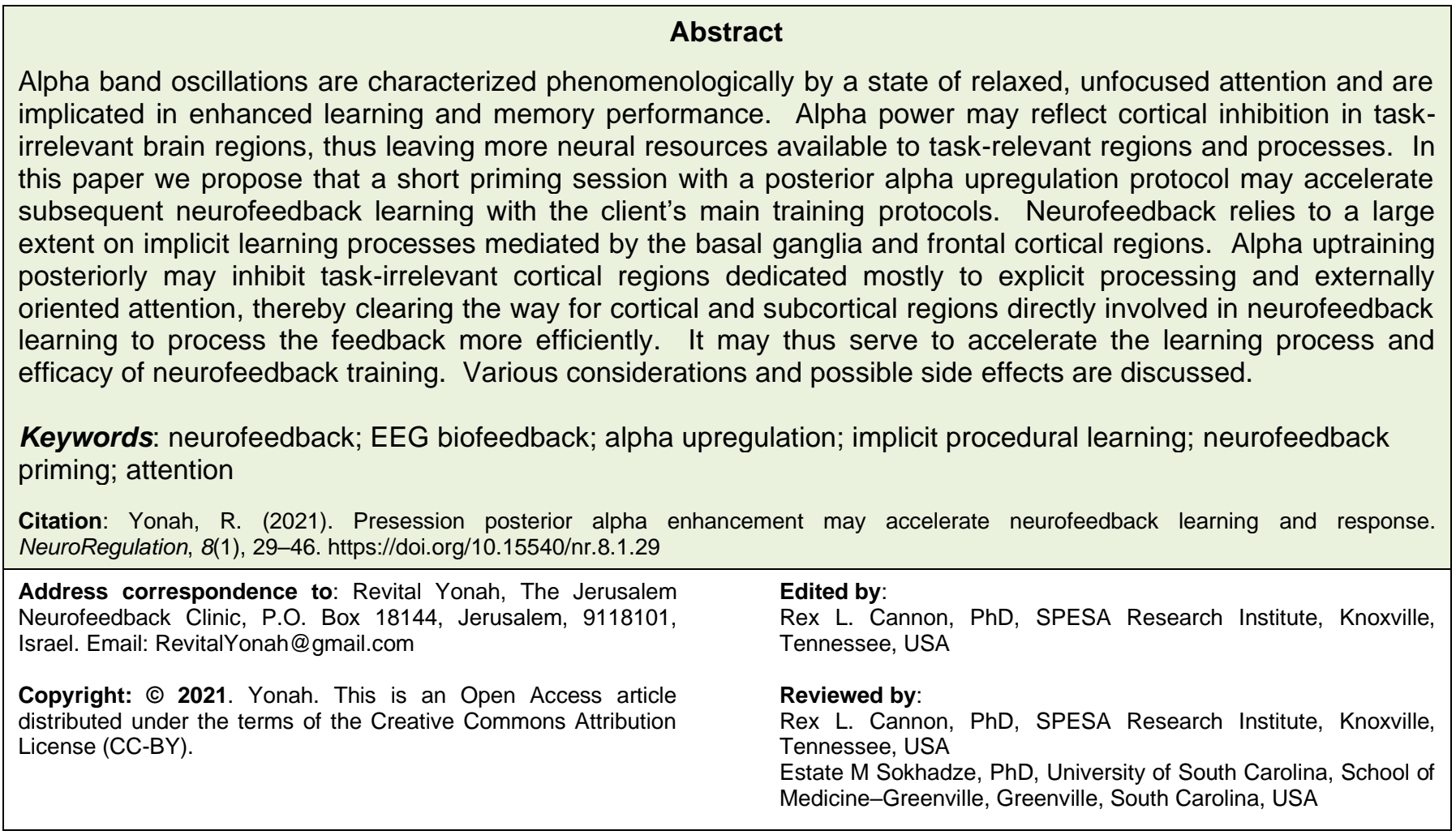

\section{Background}

Neurofeedback, a nondrug, noninvasive form of neurotherapy often used to treat attentiondeficit/hyperactivity disorder (ADHD; Moriyama et al., 2012), anxiety (Hammond, 2005; Kerson, Sherman \& Kozlowski, 2009), depression (Hammond, 2005), epilepsy (Sterman \& Egner, 2006), and other neurological and neuropsychological disorders, is based on the principles of operant conditioning of brainwave patterns (Sherlin et al., 2011; Vernon et al., 2009). In this treatment paradigm, information about brainwave activity is fed back to clients, who in turn use this information to regulate their brainwave activity and bring deviant neural activity closer to age-group norms. As a result, alleviation of symptoms may ensue, along with other cognitive, emotional, and performance gains.
Training protocols in neurofeedback are usually determined by a combination of neurometric assessment (a qEEG test and brain maps) and the client's chief complaints (Hammond, 2011). Protocols may include upregulating (enhancing) or downregulating (suppressing) different EEG frequency bands in various brain regions with the aim of bringing them closer to age-group norms. EEG bands may be standard and preset (i.e., delta: up to $4 \mathrm{~Hz}$, theta: $4-8 \mathrm{~Hz}$, alpha: $8-12 \mathrm{~Hz}$, beta: $13-$ 30 , etc.), or individually determined for each client (Bazanova \& Aftanas, 2010).

While neurofeedback is an effective, efficient treatment for a host of different neuropsychiatric disorders (Niv, 2013) and has good response rates, it is a fact that some clients do not respond to this treatment modality and are unable to self-regulate 
their brainwaves (Emmert et al., 2016; Sitaram et al., 2017), while among responders, the speed of response and effect size vary, with some clients requiring a large number of sessions to achieve significant clinical results (Barabasz \& Barabasz, 1995; Emmert et al., 2016). Accelerating the learning process in neurofeedback may prove beneficial: if clients respond to neurofeedback training faster and see clinically significant effects sooner, the chances of client retention during the critical first weeks of training increase and hence the probability of success in treatment dramatically improves.

Here we would like to suggest that presession posterior alpha upregulation may accelerate the learning process in subsequent neurofeedback training with the client's main training protocols and may therefore enhance the training efficiency and effectiveness. The main points pertaining to this proposal are as follows: alpha brainwaves have been correlated with neural inhibition of taskirrelevant cortical brain regions (Jensen \& Mazaheri, 2010; Klimesch, Sauseng, \& Hanslmayr, 2007). This neural inhibition may enhance learning and performance by reducing interference from taskirrelevant brain regions and processes, thereby allowing better information flow and allocation of neural resources to task-relevant brain regions (Jensen \& Mazaheri, 2010). While some of the evidence for the cortical inhibition theory is correlative, there is empirical evidence that it may be causative as well: causing the brain to enhance alpha with different endogenous and exogenous methods on task-irrelevant brain regions has been shown to improve performance on a variety of different cognitive tasks. Neurofeedback has been defined as procedural skill learning, a form of nondeclarative, implicit learning, which engages cortical and subcortical regions, including a significant role of the striatum of the basal ganglia (Birbaumer, Ruiz, \& Sitaram, 2013; Koralek, Jin, Long, Costa, \& Carmena, 2012; Scharnowski et al., 2015; Sulzer et al., 2013; Veit et al., 2012). By actively inhibiting posterior cortical areas that are not directly involved in the implicit task of neurofeedback learning per se, presession posterior alpha upregulation just prior to (or at the same time as) the training session with the client's main training protocols may in fact reduce interference and allow more optimal information processing by taskrelevant regions and networks during the subsequent self-regulation session. This priming protocol, when used on parietal brain regions involved in explicit cognitive processing, conscious awareness, and externally oriented attention, may elicit an open attentional state and facilitate the implicit, unconscious learning processes involved in neural self-regulation. Once clients start responding to this protocol, subsequent training with other training protocols may be facilitated and accelerated. Support from other adjunctive techniques that enhance the effect of neurofeedback training by modulating attention is also discussed.

Needless to say, alpha enhancement should only be performed if such a protocol is not contraindicated by the client's presenting symptoms and qEEG test, and when it does not clash with the client's other treatment protocols. Also, for clients who do not respond to neurofeedback at all, such priming may not help. For the nonresponders, depending on the cause of their inability to learn from neurofeedback, alternative exogenous and endogenous techniques that enhance alpha and do not rely on neurofeedback may achieve a facilitatory effect.

In what follows, we will elaborate on each of the points made above. In addition, we will also briefly discuss different variables pertaining to this proposal, namely electrode placement, individual versus standard alpha band upregulation, as well as a word of caution about possible iatrogenic effects that may result from excessive or contraindicated alpha upregulation. But let us first begin with a short description of the alpha band, its neural correlates and phenomenology.

\section{The Alpha Band Frequency}

The alpha band is nestled between the brain's slow waves (delta and theta) on the one end and fast waves (beta and gamma) on the other. While delta and theta are characterized by a state of drowsiness and daydreaming (and are prevalent in sleep), and the range of beta and gamma frequencies is characterized by a state of focused attention, high alertness, and concentration, reflecting neuronal processing (Jensen \& Mazaheri, 2010), alpha is the "bridge" between these different brain-states and is characterized by a relaxed, calm state of open, unfocused attention (Alhambra, Fowler, \& Alhambra, 1995). High alpha amplitudes may reflect an internal focus of attention (Ray \& Cole, 1985), whereas low-powered alpha may indicate externally oriented attention (Hanslmayr, Gross, Klimesch, \& Shapiro, 2011).

The alpha wave morphology is that of a sinusoidal wave (Klimesch, 1999; Kropotov, 2009) and is 
usually defined as ranging from 8 to 12 cycles per second. However, this fixed frequency band is not exhaustive of all possible alpha ranges. The alpha frequency of young, healthy adults may be somewhere between $7 \mathrm{~Hz}$ and $13 \mathrm{~Hz}$ (Klimesch et al., 2004), but a wider alpha range $(6-16 \mathrm{~Hz})$ has also been acknowledged (Bazanova \& Aftanas, 2008). The alpha rhythm is believed to be generated and modulated by both thalamocortical and corticocortical pathways (Bollimunta, Mo, Schroeder, \& Ding, 2011; Hammond, 2002).

There are different alpha rhythms in the brain, each in a different location (Kropotov, 2009). The posterior alpha rhythm, consistently localized parieto-occipitally, is the most dominant rhythm in the wakeful resting EEG in humans (Romei, Gross, \& Thut, 2010). Normally, alpha has maximal power in posterior brain regions in the wakeful resting state with eyes closed, and its power decreases dramatically when the eyes are open, to allow faster waves to engage the brain in visual information processing (Adrian \& Matthews, 1934), a phenomenon that has come to be known as alpha blocking. Posterior alpha oscillations have been found to be partly generated by areas around the calcarine fissure and secondary visual and parietal cortices (Thut, Nietzel, Brandt, \& Pascual-Leone, 2006).

The alpha rhythm has been linked to memory performance (Klimesch, Doppelmayr, Pachinger \& Ripper, 1997), problem solving (Jaušovec, 1996), internally directed attention (Cooper, Croft, Dominey, Burgess, \& Gruzelier, 2003; Ray \& Cole, 1985), creativity (Fink et al., 2009; Hardt \& Gale, 1993) and hypnotizability (Faymonville, Boly, \& Laureys, 2006), among other phenomena.

The alpha rhythm has also been associated with intelligence (Doppelmayr et al., 2005; Jaušovec, 1996), as research shows that highly intelligent people display more alpha power compared to those with average intelligence (Doppelmayr, Klimesch, Stadler, Pöllhuber, \& Heine, 2002; Doppelmayr et al., 2005). The alpha frequency is believed to reflect the speed of an individual's cognitive processing capabilities and memory performance, since it was found to significantly correlate with an individual's response times in cognitive tasks (Surwillo, 1961). Also, experimental studies show that individuals who are deemed good performers on memory tasks have an alpha frequency that is about $1 \mathrm{~Hz}$ higher than that of age-matched individuals who are less competent on such tasks (Klimesch, 1999). In addition, in adults, the power and frequency of the individual alpha band decrease with age (Hammond, 2002), and greater decreases are evident in people with mild memory impairment (Jelic et al., 2000).

Alpha is not a unitary rhythm. It is composed of several frequency bands (Klimesch et al., 2007). The alpha rhythm may be divided into two subbands: lower alpha $(8-10 \mathrm{~Hz})$, and upper alpha (10-12 Hz; Verstraeten \& Cluydts, 2002), and some researchers divide the individual alpha band further, to three different subbands (Klimesch, Doppelmayr, Russegger, Pachinger, \& Schwaiger, 1998; Wu \& Liu, 1995). Findings from empirical studies suggest that the lower alpha band is related to general, tonic attention, whereas the upper alpha band is related to memory (specifically, semantic memory), sensory processes, and a phasic (i.e., event-related) mode of attention (Capotosto et al., 2015; Klimesch, Doppelmayr, \& Hanslmayr, 2006).

A decrease in alpha power (i.e., event-related desynchronization, or ERD) is associated with active cognitive processing, whereas an increase in alpha oscillatory power (i.e., event-related synchronization, or ERS) is associated with cortical inhibition or deactivation and internally oriented brain states and attention (Cooper et al., 2003; Hanslmayr et al., 2011; Klimesch et al., 2007) and may reflect topdown control processes (Bazanova, 2012). It was demonstrated that ERD of the lower alpha band is not restricted to a certain location, but is widespread on the scalp, whereas upper alpha ERD tends to appear in more restricted cortical regions (Klimesch et al., 2006).

An important measure that is often considered in neurometric assessments is the individual alpha peak frequency (iAPF; Arns, 2012; Bazanova \& Vernon, 2014), which is the discrete frequency with the highest power within the alpha band (Angelakis et al., 2007), and as such, it is normally the most prominent rhythm in the brain in the wakeful resting state with eyes closed (Klimesch, 1999). The iAPF changes with age in an inverted u-shape fashion: low in infancy and old age and high in young adulthood and middle age (Angelakis et al., 2007), and is a little over $10 \mathrm{~Hz}$ for healthy, young adults (Klimesch, 1999). The iAPF may reflect processing speed (i.e., higher iAPF reflecting higher processing speed; Arns, 2012) while lower iAPF may be a characteristic of neurodegenerative diseases, anoxia, and age-related decline (Arns, 2012; Klimesch, 1999). The iAPF may also serve to define the individual alpha band and the individual theta 
band, and it is the anchor point between the individual lower alpha and upper alpha bands (Klimesch, 1999).

In neurofeedback, alpha may be downregulated or upregulated, depending on the client's symptoms, qEEG test and training goals. Alpha downregulation has been performed with stroke patients suffering from visuospatial neglect (Ros et al., 2017) and adults suffering from ADHD (Deiber et al., 2020) and anxiety (Kerson et al., 2009), among other disorders. Alpha upregulation may be performed to improve memory (Kober et al., 2015; Nan et al., 2012) and get clients into deeper states (as in the alpha/theta protocol) in the treatment of alcoholism, depression (Saxby \& Peniston, 1995) and posttraumatic stress disorder (PTSD; Peniston, \& Kulkosky, 1991). Posterior alpha enhancement protocols have also been associated with an induced sense of calm and pleasant relaxation (Angelakis et al., 2007; Norris, Lee, Cea \& Burshteyn, 1998), reduced stress and anxiety among highly anxious individuals (Hardt \& Kamiya, 1978) and reductions in stress response indices such as blood pressure (Norris, Lee, Burshteyn, \& Cea-Aravena, 2000). Given the correlation of alpha power with cognitive performance and processing speed, alpha upregulation, especially in the upper alpha band, is a popular protocol for peak performance and cognitive enhancement (Escolano, Aguilar, \& Minguez, 2011; Zoefel, Huster, \& Herrmann, 2011). Alpha upregulation may be performed with eyes open (Putman, 2000) or with eyes closed (Fell et al., 2002; Hardt \& Gale, 1993), and there are differing opinions among clinicians and researchers as to the most effective way to perform such training (Vernon et al., 2009).

\section{Alpha Oscillations as Cortical Inhibition}

When the brain encounters incoming stimuli, what determines how they are subsequently processed is not only the nature of each stimulus, but also the baseline neural state and ongoing neuronal dynamics in the brain at the time of stimulus presentation (Buonomano \& Maass, 2009; Scharnowski et al., 2015; von Stein \& Sarnthein, 2000). Research demonstrates that ongoing oscillatory activity before or during a perceptual event or a cognitive task influences subsequent perception and task performance (Angelakis et al., 2007; Hanslmayr, Sauseng, Doppelmayr, Schabus, \& Klimesch, 2005; Jensen \& Mazaheri, 2010). Although prestimulus alpha power may have a detrimental effect on stimulus detection under difficult perceptual conditions (Ergenoglu et al., 2004; van Dijk, Schoffelen, Oostenveld \& Jensen, 2008; see Klimesch et al., 2007 for an interpretation of this finding along the lines of the cortical inhibition hypothesis), it has been found to have an enhancing effect on learning, memory, and other complex cognitive functions (Klimesch et al., 2007). Here we will focus on the enhancing effect that alpha oscillations have on learning, memory, and cognitive performance.

The functional meaning of alpha oscillations is still debated, and several hypotheses have been put forward to explain the role of this prominent EEG rhythm. Alpha oscillations were traditionally defined as the idling rhythm of the brain, reflecting reduced sensory and cognitive processing. Support for this view came from studies showing that alpha power decreases when subjects perform a task (Pfurtscheller, Stancák, \& Neuper, 1996). However, an alternative, more recent hypothesis, suggesting that the role of alpha oscillations in the brain is to actively inhibit task-irrelevant brain regions in a topdown fashion, has been gaining ground and receiving substantial research support. Jensen and Mazaheri (2010) suggested that alpha oscillations (alpha ERS) reflect a state of cortical inhibition or deactivation which suppresses distractions from task-nonessential processes by actively inhibiting task-irrelevant brain regions. This inhibition gates information and routes it to task-relevant brain regions, a mechanism termed gating by inhibition (see also Cooper et al., 2003; Worden, Foxe, Wang, \& Simpson, 2000; van Dijk et al., 2008; but see Knyazev, Savostyanov, \& Levin, 2006). In this model, alpha reflects top-down control and is used to actively inhibit task-irrelevant processes and brain regions, thus increasing the signal-to-noise ratio and improving the efficiency of information processing and task performance.

Highly intelligent people exhibit more alpha (and therefore less cortical activation) in task-irrelevant brain regions during task performance compared with people of average intelligence. This may be due to the fact that more intelligent people may use only task-relevant brain regions while inhibiting other, task-irrelevant areas, whereas people with average intelligence may activate also taskirrelevant brain regions during task performance, which interferes with their ability to perform (Jaušovec, 1996). Thus, more efficient cognitive processing occurs when task-nonessential processes and brain regions are inhibited (Doppelmayr et al., 2005; Vernon et al., 2009). In 
support of this, alpha ERS can be seen over brain regions that are not essential for the task being performed (Klimesch et al., 2007). It is also seen in tasks in which a learned response must be withheld or inhibited, and research has demonstrated that large upper alpha ERS in a reference interval just prior to a task trial is related to large alpha ERD during the trial and to better performance (Klimesch et al., 2006; Klimesch, Doppelmayr, Röhm, Pöllhuber, \& Stadler, 2000).

There are numerous studies that demonstrate this. For example, in a word sequence memorization task, word sequences that were encoded while there was an increase in parieto-occipital alpha power were better remembered than sequences that were encoded during trials of lower-voltage posterior alpha. The authors even managed to predict which words would be remembered based on the encoding-stage posterior alpha activity alone. They attributed this to the fact that the parietal and occipital lobes are not directly needed for the cognitive processing in the word-sequence encoding stage, so an efficient inhibition of these brain regions (as reflected by increased posterior alpha power) reduces interference and thus enhances performance by allowing better processing of information in task-relevant brain regions (Meeuwissen, Takashima, Fernández, \& Jensen, 2011). In addition, Haegens and colleagues demonstrated alpha power increases in the somatosensory cortex ipsilateral to tactile stimuli in validly cued trials, which were associated with participants' increased accuracy and reaction speed (Haegens, Händel, \& Jensen, 2011). It was suggested that such an effect may have been mediated by top-down attentional control by the frontal cortex over somatosensory alpha activity, that caused the disengagement of task-irrelevant regions (Haegens, Osipova, Oostenveld, \& Jensen, 2010).

Similarly, in tasks that project stimuli in one visual hemifield at a time, alpha ERD appears in the hemisphere contralateral to the cued and attended hemifield, and at the same time, alpha ERS appears in the ipsilateral hemisphere (Kelly, Lalor, Reilly \& Foxe, 2006; Worden et al., 2000). The functional meaning of this phenomenon may be that, with alpha ERD, the contralateral hemisphere is activated to process the stimulus, whereas in the ipsilateral hemisphere, alpha ERS inhibits task-irrelevant brain regions to suppress distracting stimuli and processes in order to allow for better information flow to the task-relevant hemisphere (Thut et al., 2006). The same principle seems to hold true also when it comes to brain regions dedicated to different sensory modalities. While performing tasks that require allocation of attention to one sensory modality, task-nonessential brain regions dedicated to the processing of another sensory modality show increased alpha power (Foxe, Simpson, \& Ahlfors, 1998) and decreased regional cerebral blood flow (Haxby et al., 1994; Kawashima, O'Sullivan, \& Roland, 1995), which reflects functional inhibition, thus leaving more resources available to the taskrelevant brain regions to perform the task. Even within the same sensory modality, this dichotomy of brain activation in task-relevant regions and deactivation in task-irrelevant brain regions occurs. For example, when subjects direct their attention to a linguistic task, decreased activation is found in unrelated brain regions dedicated to the processing of motion stimuli (Rees, Frith, \& Lavie, 1997). Lastly and of importance to our proposal, Ray and Cole (1985) found parietal alpha increases during cognitive and emotional tasks that did not require attention to the external environment, thus permitting more efficient processing of internal tasks.

Correlation, however, should not be equated with causation. Demonstrating that increased alpha activity in task-irrelevant brain regions improves learning and task performance does not necessitate that causally increasing alpha power will do the same. To prove causality, a few studies were conducted, demonstrating that causally enhancing alpha power in task-irrelevant brain regions may indeed improve performance. For instance, Sauseng and colleagues enhanced alpha $(10 \mathrm{~Hz})$ power causally with repetitive transcranial magnetic stimulation (rTMS) over the posterior parietal cortex ipsilateral to visual items to be retained in memory and found that it enhanced short-term visual memory performance, whereas a similar treatment on homologous brain regions contralaterally actually hampered performance on the task. This effect was specific to the $10 \mathrm{~Hz}$ stimulation over parietal brain regions (Sauseng et al., 2009). Similarly, Lustenberger and colleagues demonstrated that transcranial alternating current stimulation (tACS) to enhance frontal alpha activity bilaterally during a divergent thinking test enhanced creativity. This effect was specific to the alpha rhythm $(10 \mathrm{~Hz})$, as tACS at $40 \mathrm{~Hz}$ did not yield similar results (Lustenberger, Boyle, Foulser, Mellin, \& Fröhlich, 2015). This may mean that inhibition of the rational, critical thinking frontal brain is conducive to original thinking and ideation and that it prevents internal information processing being disturbed by external stimuli (Fink, Grabner, Benedek, \& Neubauer, 2006). 
Evidence for the causal role of alpha oscillations in improving performance were obtained also through studies utilizing neurofeedback alpha upregulation protocols to improve performance on different tasks. For example, Bazanova, Verevkin, and Shtark (2007) found that musicians who underwent neurofeedback training to increase their upper alpha power demonstrated both increased alpha power and improved musical performance. Also, Hanslmayr and colleagues reported that using neurofeedback training to enhance the individual upper alpha band resulted in improved performance on a mental rotation task after only one session (Hanslmayr et al., 2005). Both music performance and mental rotation are skills that depend on inner control of information processing (Alexeeva, Balios, Muravlyova, Sapina, \& Bazanova, 2012). These findings demonstrate how causally increasing alpha power may improve performance on such tasks. It appears that higher levels of baseline alpha power allow better flexibility in inhibiting (or not inhibiting) different processes, according to the needs of the task (Vernon et al., 2009).

Neurofeedback is considered to be a form of nondeclarative, implicit learning, with a critical role of the striatum of the basal ganglia (Birbaumer et al., 2013; Emmert et al., 2016; Koralek et al., 2012). In what follows, we suggest that presession posterior alpha upregulation may help to relatively deactivate posterior cortical regions that are not directly involved in the implicit aspects of neurofeedback learning, thus decreasing interference from tasknonessential processes and leaving more neural resources available to other brain regions and networks relevant to self-regulation learning. This may accelerate response to neurofeedback with the client's main treatment protocols and may also increase effect size. This may be compatible with both the "alpha as idling" and "alpha as cortical inhibition" hypotheses. But before we discuss this suggestion, let us briefly explore the difference between implicit and explicit types of learning and their neural correlates.

\section{Implicit Versus Explicit Learning}

The brain is a learning apparatus. There are many different types of learning, and while they can be divided and grouped by different criteria, one of the most basic divisions is between explicit, declarative learning and implicit, nondeclarative learning (Squire \& Zola, 1996). Explicit learning is the kind of learning that learners have conscious awareness of, so that they can think about what they learned and articulate it to themselves and to others. Tulving (1972) divided explicit memory into episodic memory (i.e., memory of personal events) and semantic memory (i.e., memory of facts and common knowledge). Implicit (nondeclarative) learning, on the other hand, is achieved unconsciously and is hard to verbalize to others (and even to oneself). That is, implicit learning involves the acquisition of tacit knowledge which is inaccessible to conscious awareness and is expressed through performance (Reber \& Squire, 1994).

Explicit and implicit learning exhibit different neural correlates and rely on qualitatively distinct neural systems (Rugg et al., 1998), with explicit memory relying more on the medial temporal lobe (the hippocampus; Eichenbaum, 1999) along with frontal and parietal cortices (Yang \& Li, 2012), and certain types of implicit memory relying more on subcortical structures such as the striatum of the basal ganglia (Heindel, Salmon, Shults, Walicke, \& Butters, 1989; Poldrack et al., 2001) and engaging fronto-striatal networks (Yang \& Li, 2012). It was demonstrated that during implicit inferences, prefrontal regions deactivate parietal networks involved in externally oriented attention (which appears as prefrontal alpha ERD and parietal alpha ERS), while during tasks involving explicit learning and memory, the opposite pattern (i.e., prefrontal alpha ERS and parietal alpha ERD) can be seen (Wokke \& Ro, 2019). It is important to note, however, that implicit and explicit learning systems may overlap and share a significant portion of the same networks (Yang \& Li, 2012) and that, very often, tasks may rely on both systems (Destrebecqz et al., 2005), such as when one learns to play music (Rohrmeier \& Rebuschat, 2012) or acquires a new language (Peigneux, Laureys, Delbeuck, \& Maquet, 2001).

One illuminating demonstration of the functional and anatomical division between implicit and explicit learning is the double dissociation between these two types of learning in different patient populations (Packard \& Knowlton, 2002). For instance, people with amnesia, who have a temporal lobe dysfunction, tend to perform well in tasks requiring implicit inferences but not in tasks requiring explicit learning and memory (Graf, Squire, \& Mandler, 1984; Milner, Corkin, \& Teuber, 1968), whereas Parkinson's disease patients, who have basalganglia dysfunction, display the opposite pattern (Knowlton, Mangels, \& Squire, 1996).

Implicit, nondeclarative learning is not a single entity, but rather a set of heterogenous phenomena (Seger, 
Prabhakaran, Poldrack, \& Gabrieli, 2000). There are different types of implicit learning, and they rely on different cortical and subcortical circuits. Under the broad category of implicit learning are different types of learning, such as: procedural learning (skills and habits), instrumental learning (reinforcement learning or operant conditioning), perceptual learning, priming, classical conditioning, classification learning, probability learning, artificial grammar learning, and more (Heindel et al., 1989; Reber \& Squire, 1994; Seger et al., 2000; Sigala, Haufe, Roy, Dinse, \& Ritter, 2014; Squire \& Zola, 1996). Each of these engages different cortical and subcortical brain regions.

Procedural learning is the learning of automatic skills and sensorimotor habits which are mostly unconscious, such as when one learns how to juggle three or more balls or ride a bicycle (Baars \& Gage, 2010). The dorsal striatum (comprised of the putamen and caudate) functions as the basal ganglia's interface to the cortex (Zotev, Misaki, Phillips, Wong, \& Bodurka, 2018) and is implicated in procedural skill learning (Squire \& Zola, 1996). The striatum of the basal ganglia has also been implicated in feedback learning (Grahn, Parkinson \& Owen, 2008; Peters \& Crone, 2017), stimulus response learning (Packard \& Knowlton, 2002), and instrumental learning (Liljeholm \& O'Doherty, 2012). Neurofeedback involves these types of learning and, as we will see next, it can be viewed as a complex type of nondeclarative, procedural learning.

\section{Neurofeedback as a Form of Implicit Learning}

Neurofeedback has been defined as a form of procedural skill learning (Kober, Witte, Ninaus, Neuper, \& Wood, 2013; Sitaram et al., 2017) and instrumental learning (Gruzelier \& Egner, 2004), and as such, it may not be an explicit conscious process, but rather an implicit type of learning (Birbaumer et al., 2013). Participants in neurofeedback training can recognize mental states and body sensations and use these to infer their brain states, but they do not have direct conscious knowledge of the neurophysiological activity in their brains per se. Indeed, it has been suggested that the role of explicit learning mechanisms in neurofeedback may be limited (Kober et al., 2013). In addition, the basal ganglia have been shown to be involved in skill learning (Squire \& Zola, 1996) and instrumental learning (Yin \& Knowlton, 2006), and studies of functional magnetic resonance imaging (fMRI) neurofeedback have shown the consistent involvement of the basal ganglia in neurofeedback learning as well (Emmert et al., 2016; Lawrence et al., 2014; Scharnowski et al., 2015; Sulzer et al., 2013; Veit et al., 2012).

The term implicit learning in the context of neurofeedback may be somewhat misleading. Implicit learning does not necessarily mean that the learning occurs without any conscious awareness. Rather, implicit learning involves conscious input that is processed with implicit inferences. It is these inferences and computations that are performed without conscious awareness (Baars \& Gage, 2010). While some aspects of the neurofeedback training are conscious (i.e., the sensory feedback, the training room, the presence of the practitioner, and even some mental states and overt cognitive strategies during session), what constitutes the actual learning, that is, the inferences made by the brain concerning the neuronal activity that is being rewarded or inhibited (and the computations and physiological changes that have to be made to enhance or suppress such activity), is unconscious and constitutes implicit, nondeclarative learning. The fact that small children (Mohagheghi et al., 2017), toddlers (Cannon, Strunk, Carroll, \& Carroll, 2018), and even animals (Kobayashi, Schultz, \& Sakagami, 2010; Sterman, 1977) can successfully attain brainwave self-regulation in neurofeedback attests to the fact that neurofeedback learning is based on implicit processes. Indeed, operant conditioning of one single cortical neuron in an unanesthetized animal was shown to be feasible (Fetz, 1969), and successful neurofeedback learning was attained unconsciously by participants who were unaware that they were being trained and who had no intent to learn (Ramot, Grossman, Friedman, \& Malach, 2016). In support of this, there is ample evidence that trying to employ explicit strategies during neurofeedback training may actually hamper the attainment of self-regulation (Wood, Kober, Witte, \& Neuper, 2014), whereas having no conscious strategies during training may facilitate such learning (Kober et al., 2013; Sitaram et al., 2017; Witte, Kober, Ninaus, Neuper, \& Wood, 2013; but see: Hardman et al., 1997). It may be that the use of overt mental strategies to try to consciously control the feedback leads to a cognitive overload, and this overload interferes with the neurofeedback learning and impedes it (Kober et al., 2013). A similar suggestion was made for other types of implicit learning as well (Chafee \& Crowe, 2017; Packard \& Knowlton, 2002). 


\section{Alpha Upregulation to Facilitate the Efficiency and Efficacy of Neurofeedback}

In light of the inhibitory role that alpha oscillations have been shown to play at task-irrelevant brain regions (i.e., reducing interference and increasing the signal-to-noise ratio in the brain) and due to the fact that neurofeedback learning can be achieved unconsciously, relying on implicit learning mechanisms that are mediated to a large extent by subcortical structures such as the basal ganglia (along with other cortical and subcortical regions), it seems plausible to suggest that presession alpha upregulation at posterior electrode sites may accelerate subsequent neurofeedback learning with the client's main training protocols. The posterior (parietal and occipital) brain regions are known to be involved in vision, sensory perception integration (Konen \& Haggard, 2014), spatial cognition, motor planning (Freedman \& Ibos, 2018), visual imagery (McNorgan, 2012), conscious cognitive processing, and externally oriented attention (Wokke \& Ro, 2019). Enhancing alpha power over these areas may relatively deactivate these brain regions that are not directly involved in the neurofeedback learning per se, thus allowing task-relevant brain regions to receive and process the feedback information with less interference. This may enhance the brain's ability to perform the brainwave self-regulation more efficiently and effectively. This proposal may be valid also when considering the "alpha as idling" hypothesis: idling of task-irrelevant brain regions may leave more cognitive and neural resources available to task-relevant regions and cause less distractions and interference from tasknonessential processes.

In support of this, Wood and coauthors suggest that suppressing conscious cognitive processes that are not necessary for neurofeedback is a key to improving self-regulation in neurofeedback (Wood et al., 2014). Similarly, Ninaus et al. (2013) suggested that during neurofeedback, top-down control of taskirrelevant brain regions takes place to reduce interference from such networks while the taskrelevant networks perform the neurofeedback learning. Also, Rauch et al. (1997) suggested that in implicit learning, the striatum of the basal ganglia relieves corresponding cortical areas of their computational load, which could probably reflect the parieto-striatal dynamics during neurofeedback as well, since the striatum has neural connections with different regions in the parietal cortex (Cavanna \& Timbler, 2006; Jarbo \& Verstynen, 2015; Liljeholm \& O'Doherty, 2012).
In their meta-analysis, Emmert and colleagues found consistent basal-ganglia and frontal activations during neurofeedback across the different studies that they surveyed (Emmert et al., 2016), and this confirms the important role of these regions in neurofeedback processing. In fact, in Ninaus and colleagues' study of the neural correlates of "neurofeedback" done with sham feedback only, the authors did not find any basal-ganglia activations, possibly reflecting the fact that no real feedback was given, therefore no real learning was taking place (Ninaus et al., 2013).

While the role of the basal ganglia in the implicit processes involved in neurofeedback may be established, the picture is less clear as to the role of posterior brain regions in such learning. Different studies have reported different, sometimes contradicting, patterns of parietal activations and deactivations during neurofeedback (Emmert et al., 2016; Haller, Birbaumer \& Veit, 2010; Lam et al., 2020; Scharnowski et al., 2015). These differing patterns may be the result of the different target regions of interest (ROIs) focused on in these studies, as well as the different experimental and baseline conditions, modalities and forms of feedback and the instructions given to participants. They may also be the result of the different types of mental imagery used by participants (McNorgan, 2012). The parietal lobes were found to be engaged in explicit processing and externally oriented attention, and inhibited under internal implicit control through processes of executive control by the frontal lobes (Wokke \& Ro, 2019). Also, alpha increases were found in the parietal lobes during tasks that do not require attention to the external environment (Ray \& Cole, 1985). We would have therefore expected to see parietal deactivations during neurofeedback. However, the fact is that in some of these studies (see meta-analysis by Emmert et al., 2016) dorsal parietal activations were reported along with deactivations of areas in the medial portions of the parietal lobe (i.e., the precuneus and posterior cingulate cortex, PCC) that make up the main posterior node of the default mode network (DMN; Zotev et al., 2018). This pattern of activations and deactivations could perhaps be explained by the fact that, in these studies, participants employed overt, explicit cognitive strategies trying to consciously control the feedback by exerting cognitive effort. This means that they focused their attention intently on the external sensory feedback in an attempt to consciously, explicitly control it, rather than letting go, keeping an open focus and allowing their brain to process the neural feedback implicitly. It has 
been shown that exerting cognitive effort in goaldirected, attention-demanding tasks deactivates the precuneus and PCC (Raichle et al., 2001). It could be, therefore, that the pattern of activity revealed in Emmert et al.'s meta-analysis is a result of the explicit mental strategies and conscious, deliberate cognitive effort employed by the participants to control the feedback. In support of this, posterior brain regions involved in attention were found to be activated in explicit processing (Aizenstein et al., 2004), and the posterior parietal cortex was suggested to be involved in executive control processes during neurofeedback (Sitaram et al., 2017). Also, Veit et al. (2012) found parietal cortical activations for task conditions in neurofeedback that demanded more cognitive effort. Therefore, it may be cautiously conjectured that posterior cortical regions are not inherently involved in the implicit aspects of neurofeedback but may rather be more related to explicit cognitive strategies employed by some participants in an attempt to consciously control the external sensory feedback.

Interestingly, Scharnowski et al. (2015) found that during fMRI neurofeedback on different ROls and in opposing directions (i.e., upregulation and downregulation of the blood-oxygenation-leveldependent, or BOLD, signal), there was unexplainable consistent deactivation of the superior and ventral visual cortices in the occipital lobe, bilaterally, even though the visual displays in the training and baseline blocks were identical. It is possible that in order to learn the neurofeedback task efficiently, the brain relatively deactivated posterior brain regions as a means to suppressing the processing of irrelevant visual information.

Further support for this idea comes from Lam et al. (2020), who found that successful learners of fMRI neurofeedback were those participants who showed greater fronto-striatal activation and decreased temporo-occipital-cerebellar activation during a response inhibition task, presumably reflecting better top-down cognitive control abilities. Similarly, an association between the ability to inhibit taskirrelevant brain regions and success in kinesthetic motor imagery fMRI neurofeedback was found by Chiew, LaConte, and Graham (2012). These findings indicate that top-down attentional and executive control processes are related to better self-regulation ability in neurofeedback (Lam et al., 2020). Indeed, in accordance with this and in line with our suggestion regarding the facilitatory effect of posterior alpha upregulation, LORETA neurofeedback parietal alpha training was found to have a positive effect on executive functions (Cannon, 2012) and LORETA neurofeedback with an alpha upregulation protocol at the precuneus was found to be related to novel learning and improvements in self-regulation, in the broader sense of the word (Cannon et al., 2014). In addition, alpha activity indices were suggested to be related to self-regulation ability (Bazanova, 2012; Bazanova, Kondratenko, Kondratenko, Mernaya \& Zhimulev, 2007).

While the above-mentioned findings of the neural correlates of neurofeedback were found using fMRIneurofeedback, it is likely that a common brain network is involved in the regulation process itself (Emmert et al., 2016) and that several aspects of neurofeedback training are constant, regardless of the method used (Ninaus et al., 2013), and may therefore be at work also in EEG-neurofeedback.

\section{Alpha and the Facilitatory Power of Attention}

The alpha upregulation priming suggested here involves attentional changes. Similar attentional changes induced by other methods have been shown to accelerate neurofeedback learning. One such attentional technique that has been used to enhance neurofeedback success is a computerassisted mindfulness instruction just prior to a neurofeedback session (Da Costa, Bicho, \& Dias, 2019, 2020). Chow and colleagues showed that mindfulness meditation is associated with an increased global full-band alpha amplitude, comparable to that achieved in an alpha upregulation neurofeedback session (Chow, Javan, Ros, \& Frewen, 2017), which suggests that the facilitatory effect of mindfulness on neurofeedback may be mediated by increases in the alpha band power. Similarly, Stieger et al. (2021) demonstrated that mind-body awareness training increased participants' alpha power during rest and also accelerated their subsequent brain-computer interface $(\mathrm{BCl})$ learning, a form of open-looped neurofeedback. Findings of accelerated $\mathrm{BCl}$ learning following mind-body awareness training were reported also by Cassady, You, Doud, and He (2014). In addition, Kober et al. (2017) demonstrated that people who are regularly involved in a spiritual practice, such as prayer, learn brainwave self-regulation faster than people who rarely pray. The authors suggest that people who regularly engage in a spiritual routine are more skillful at gating and routing incoming information about their brainwaves during neurofeedback and thus manage to avoid distracting, task-irrelevant 
thoughts (and hence task-irrelevant neuronal processing). Another adjunctive attentional technique suggested to be facilitatory of subsequent neurofeedback training is the Open Focus technique (Fehmi \& Robbins, 2008), which proposes that when clients keep their attention open, without narrowing or focusing it on anything in particular, their alpha power increases and their subsequent neurofeedback training accelerates and improves dramatically. In line with this, it was found that, in alpha upregulation neurofeedback, participants who succeeded in enhancing their alpha power were those who kept an open focus and did not pay too much attention to the feedback tone (Biswas \& Ray, 2019).

The facilitatory effects that the above cited studies found were demonstrated with an alpha or sensorimotor rhythm (SMR) uptraining neurofeedback protocol. However, an attentionmodulating technique that primed and accelerated neurofeedback with different frequency bands (theta and beta) was also reported, which suggests that the priming power of attention may not be restricted to protocols enhancing the alpha band or related rhythms alone. Barabasz and Barabasz demonstrated that their Instantaneous Neuronal Activation Procedure (INAP), a short alert hypnosis procedure lasting as little as $45-90$ seconds just prior to neurotherapy sessions with a theta suppression and beta enhancement protocol, yielded similar or better results than neurotherapy alone and did this in less than half the number of sessions (Barabasz \& Barabasz, 1995, 1999). Although these researchers found that INAP was related to increased frontal beta $(14-20 \mathrm{~Hz})$, independent of neurofeedback, this technique, based on the principles of hypnosis, achieves its effect by manipulating attention networks in the brain and may therefore be related to other EEG correlates of attention as well, such as an increased alpha power. Indeed, alpha power was found to be associated with hypnotic states (Williams \& Gruzelier, 2001) and hypnotizability (Faymonville et al., 2006; London, Hart, \& Leibovitz, 1968).

\section{A Word of Caution - \\ Possible Side-Effects of Alpha Enhancement}

Overtraining with any protocol is not recommended, and extra care should be taken when it comes to protocols enhancing local synchrony in slow (i.e., alpha and theta) oscillations (Sherlin, 2009). Caution should be exercised especially when performing neurofeedback with anxious or emotionally unstable individuals using an alpha enhancement protocol (Hammond, Stockdale, Hoffman, Ayers, \& Nash, 2001) to avoid phenomena of unintended emotional release. Also, long sessions may cause clients to become drowsy and fatigued (Vernon et al., 2009), and this, in turn, may negatively affect alpha power (Biswas \& Ray, 2019). Research has shown that after as little as 2 or 3 minutes of neurofeedback training, participants managed to increase their alpha band amplitude (Travis, Kondo, \& Knott, 1974, as cited in Vernon et al., 2009), which means that short presession training of alpha upregulation may be a safe place to start. However, 10-min presession blocks may be more effective (Ancoli \& Kamiya, 1978), and depending on the client's response, longer durations (20 minutes or more) may be plausible as well (Vernon et al., 2009).

The decision of whether to perform presession alpha upregulation should be made based on the client's qEEG test and presenting symptoms and should be avoided in cases in which such a protocol is contraindicated. For example, if a client's alpha power is high compared to age-group norms, enhancing alpha further should be avoided. Also, while alpha upregulation is normally associated with a sense of calm and relaxation, for some clients it may be accompanied with increased agitation, irritability, hyperarousal, and impatience. Some may experience sleep-onset delays (Bednár, 2018). Others may be disoriented and unfocused following alpha enhancement, and in some cases alpha uptraining may enhance feelings of anxiety (Thompson \& Thompson, 2009) and temporary sadness (Ibric \& Davis, 2007). Therefore, performing short priming sessions with an alpha upregulation protocol and increasing their duration gradually while watching the client's response would be a safe way to go about it. Also, since we do not know with certainty right from the outset how each of the clients' main training protocols would affect them, we may not want to accelerate learning too fast too soon.

\section{Summary and Discussion}

Neurofeedback is a relatively safe neurotherapy method that offers clients abundant clinical, cognitive and overall well-being advantages. However, one of the main deterring points for clients who consider whether to begin neurofeedback or not is the average time it normally takes to achieve a lasting, significant clinical effect. An average neurofeedback training series may consist of 40 to 
80 sessions, which, at a rate of twice or three times a week, can take between 6 and 8 months (Barabasz \& Barabasz, 1999). In addition, especially when it comes to small children, 6 to 8 months to get the desired clinical results is a relatively long period of time and, when the effects of the training start to manifest, parents sometimes attribute the changes to the natural maturation of their child. Finding a way to accelerate neurofeedback learning would yield results faster in a way that would help keep clients committed to the process and deem the maturation misjudgment in the case of children implausible (Barabasz \& Barabasz, 1995).

In this paper we suggested that the learning process in neurofeedback training may be facilitated and accelerated by a short presession training with an alpha enhancement protocol over posterior brain regions. As shown here, the basal ganglia are directly involved in neurofeedback learning along with other cortical and subcortical regions. Achieving relative deactivation of posterior cortical regions dedicated to externally oriented attention and explicit sensory and cognitive processing through alpha uptraining may allow information to flow with better efficiency to the task-relevant regions involved in neurofeedback, where it would be subsequently processed with less interference and better effectiveness.

The presession alpha upregulation priming can be performed in different ways, and the training variables may vary between clients. For each client, clinicians should determine such training variables as: the duration of the presession alpha upregulation training; whether the protocol should utilize the standard alpha band or the individual alpha band (Bazanova, 2012; Bazanova \& Aftanas, 2010); the lower alpha (reflective of an unaware state of relaxation), the upper alpha (reflective of an alert state and an open awareness with no focus on anything in particular in the external or internal environment; Sherlin, 2009), or the entire alpha band; whether the presession alpha training should be performed with eyes open or eyes closed (Vernon et al., 2009); as a single-channel protocol or with more than one channel (Sherlin, 2009); the exact electrode site (parietal, occipital, or another); just prior to the beginning of the session or at the same time as the client's main training protocols (if the main protocols involve a posterior placement), etc. In the question of standard versus individual alpha band, it seems that the latter is preferred, as it reflects the client's functional alpha more faithfully
(Bazanova, 2012; Klimesch, 1999) and research has shown that training with the client's individual alpha is more effective than training with the preset, standard alpha band (Bazanova \& Aftanas, 2010). In addition, since the individual alpha frequency changes with age, when the clients are young children, their individual alpha frequency may be much lower than the standard $8-12 \mathrm{~Hz}$ band (Niedermeyer, 1999). In terms of the electrode site to be trained, it seems that posterior placements are good candidates, for all the reasons detailed above, namely, the role of these cortices in externally oriented attention and explicit cognitive and sensory processing. Alpha at central sites is related to the SMR band (Kropotov, 2009) and tends to be accompanied by motor inhibition and reduced sensorimotor interference. However, SMR upregulation is related to enhanced externally oriented attention, as it was shown to improve performance in tasks such as shooting (Gong, Nan, Yin, Jiang, \& Fu, 2020) and gulf putting (Cheng et al., 2015). Therefore, it seems that alpha enhancement in posterior sites is the preferred option, due to these regions' role in externally oriented attention and explicit cognitive and sensory processing. Experience shows that parietal alpha enhancement with eyes open may be effective. In terms of the training montage, one-channel referential alpha upregulation may lead to transient phenomena of emotional imbalance among vulnerable individuals, which is the reason why some researchers and clinicians prefer to start with either a bipolar protocol or a two-, four- or multichannel alpha synchrony training (Sherlin, 2009).

Not in every case is a presession priming with an alpha upregulation protocol indicated. Some clients may exhibit excessive alpha power, which may be the case, for example, in some subtypes of ADHD or in depression (Byeon, Choi, Won, Lee, \& Kim, 2020). Also, alpha upregulation should be avoided when there is a reason to believe that it may clash with the client's other training protocols or overall training goals. However, in cases when there is no obvious reason to decide against alpha enhancement, alpha upregulation should be considered as an adjunctive, priming protocol to be performed just prior to neurofeedback sessions. This protocol affects the client's attentional networks and thus may increase the brain's receptivity to subsequent brainwave self-regulation training. Still, for some clients, this protocol may be accompanied by iatrogenic effects, so it is recommended to start with short periods of presession alpha upregulation 
and to gradually increase their duration according to the client's reaction and treatment response.

To establish this suggestion empirically, controlled, large-sampled studies should be conducted, comparing the speed, efficacy, and effect size of brainwave self-regulation training with and without the priming effect of presession posterior alpha upregulation. Such studies should be performed with different training protocols following the presession training. They should be conducted with a sample of healthy subjects as well as with specific clinical populations (preferably divided by electroencephalographic endophenotypes). In addition, research should try to determine what may be the most efficient way to conduct such priming sessions in terms of the different variables outlined above.

Some clients are deemed nonresponders to neurofeedback training, and trying to prime their brain with a neurofeedback protocol may prove futile, for obvious reasons. Depending on the reasons for this inability to learn self-regulation through neural feedback (and these may vary between clients and may be cognitive, emotional, or physiological in nature), it may be that perhaps at least some of these clients would respond to neurofeedback if their brain is driven by other means, exogenous or endogenous, to produce cortical alpha brainwaves. This, in turn, may decrease resistance, modulate attention, inhibit taskirrelevant cortical regions and increase the signal-tonoise ratio, and thus clear the way for task-relevant regions to take the lead role during the subsequent neurofeedback session. This may be done just prior to the neurofeedback session (or during the session) with techniques such as audio-visual entrainment (AVE; Collura \& Siever, 2009), rhythmic visual stimulation ("flicker"; Gulbinaite, van Viegen, Wieling, Cohen, \& VanRullen, 2017), cranial electrotherapy stimulation (CES; Kirsch \& Nichols, 2013), binaural beats (Foster, 1990) and various other brain-driving methods mentioned earlier. Even though the effects of these methods are not always apparent as alpha in posterior regions, they may induce a sense of calm and modulate attention in a way that would prove beneficial for subsequent neurofeedback training. Since the use of some of these techniques is not always feasible for every client (or for every clinician), it may also be the case that readily available endogenous, attention modulating techniques would achieve a facilitatory effect; for example, autogenic training, breathing exercises (Green \& Green, 1977), mindfulness meditation (Da
Costa et al., 2019, 2020), relaxation, meditating on a positive affirmation, effortless mental imagery such as pleasant scenes (Foster, 1990), and keeping an open focus without thinking of anything in particular (Fehmi \& Robbins, 2008). In cases in which the clinicians are licensed hypnotherapists, the INAP (alert hypnosis) technique (Barabasz \& Barabasz, 1995) may prove useful as well. The key point is to induce attentional changes that will allow implicit learning processes to take place during neural selfregulation with minimal interference from externally oriented attentional networks.

\section{Author Disclosure}

The author does not have any grants, financial interests or conflicts to disclose.

\section{References}

Adrian, E. D., \& Matthews, B. H. C. (1934). The Berger rhythm: Potential changes from the occipital lobes in man. Brain, 57, 355-385. https://doi.org/10.1093/brain/awp324

Aizenstein, H. J., Stenger, V. A., Cochran, J., Clark, K., Johnson, M., Nebes, R. D., \& Carter, C. S. (2004). Regional brain activation during concurrent implicit and explicit sequence learning. Cerebral Cortex, 14(2), 199-208. https://doi.org /10.1093/cercor/bhg119

Alexeeva, M. V., Balios, N. V., Muravlyova, K. B., Sapina, E. V., \& Bazanova, O. M. (2012). Training for voluntarily increasing individual upper alpha power as a method for cognitive enhancement. Human Physiology, 38(1), 40-48. https://doi.org/10.1134/S0362119711060028

Alhambra, M. A., Fowler, T. P., \& Alhambra, A. A. (1995). EEG biofeedback: A new treatment option for ADD/ADHD. Journal of Neurotherapy, 1(2), 39-43. https://doi.org/10.1300 /J184v01n02_03

Ancoli, S., \& Kamiya, J. (1978). Methodological issues in alpha biofeedback training. Biofeedback and Self-Regulation, 3, 159-183. https://doi.org/10.1007/BF00998900

Angelakis, E., Stathopoulou, S., Frymiare, J. L., Green, D. L., Lubar, J. F., \& Kounios, J. (2007). EEG neurofeedback: A brief overview and an example of peak alpha frequency training for cognitive enhancement in the elderly. The Clinical Neuropsychologist, 21(1), 110-129. https://doi.org/10.1080 /13854040600744839

Arns, M. (2012). EEG-based personalized medicine in ADHD: Individual alpha peak frequency as an endophenotype associated with nonresponse. Journal of Neurotherapy, 16(2), 123-141. https://doi.org/10.1080/10874208.2012.677664

Baars, B. J., \& Gage, N. M. (2010). Learning and memory. In B. J. Baars \& N. M. Gage (Eds.), Cognition, brain, and consciousness (2nd ed., pp. 304-343). Cambridge, MA: Academic Press. https://doi.org/10.1016/B978-0-12-3750709.00009-7

Barabasz, A. F., \& Barabasz, M. (1995). Attention deficit hyperactivity disorder: Neurological basis and treatment alternatives. Journal of Neurotherapy, 1(1), 1-10. https://doi.org/10.1300/J184v01n01_01

Barabasz, A. F., \& Barabasz, M. (1999). Treating ADHD with hypnosis and neurotherapy. Paper presented at the Annual Convention of the American Psychological Association. Boston, MA. Retrieved from https://files.eric.ed.gov/fulltext /ED435076.pdf 
Bazanova, O. M. (2012). Alpha EEG activity depends on the individual dominant rhythm frequency. Journal of Neurotherapy, 16(4), 270-284. https://doi.org/10.1080 $/ 10874208.2012 .730786$

Bazanova, O. M., \& Aftanas, L. I. (2008). Individual measures of electroencephalogram alpha activity and non-verbal creativity. Neuroscience and Behavioral Physiology, 38(3), 227-235. https://doi.org/10.1007/s11055-008-0034-y

Bazanova, O. M., \& Aftanas, L. I. (2010). Individual EEG alpha activity analysis for enhancement neurofeedback efficiency: Two case studies. Journal of Neurotherapy, 14(3), 244-253. https://doi.org/10.1080/10874208.2010.501517

Bazanova, O. M., Kondratenko, A., Kondratenko, O., Mernaya, E., \& Zhimulev, E. (2007, June). New computer-based technology to teach peak performance in musicians. Presented at the 29th International Conference on Information Technology Interfaces, Cavtat, Croatia, Vol. 7, pp. 39-44. Retrieved from https://www.researchgate.net /publication/4266106_New_Computer-

Based_Technology_to_Teach_Peak_Performance_in_Musici ans

Bazanova, O. M., Verevkin, E. G., \& Shtark, M. B. (2007). Biofeedback in optimizing psychomotor reactivity: II. The dynamics of segmental alpha-activity characteristics. Human Physiology, 33(6), 695-700. https://doi.org/10.1134 IS0362119707060059

Bazanova, O. M, \& Vernon, D. (2014). Interpreting EEG alpha activity. Neuroscience \& Behavioral Reviews, 44, 94-110. https://doi.org/10.1016/j.neubiorev.2013.05.007

Bednár, J. (2018, September 7). Hacking the brain with opensource EEG and neurofeedback (Beyond Psychedelics 2018 talk) [Video]. YouTube. https://www.youtube.com /watch?v=C tS3cEfp9o\&list=PLd058iT9keO5maJC7bxmcQq enyEMf7EQr\&index $=6 \& \mathrm{t}=1219 \mathrm{~s}$

Birbaumer, N., Ruiz, S., \& Sitaram, R. (2013). Learned regulation of brain metabolism. Trends in Cognitive Sciences, 17(6), 295-302. https://doi.org/10.1016/j.tics.2013.04.009

Biswas, A., \& Ray, S. (2019). Alpha neurofeedback has a positive effect for participants who are unable to sustain their alpha activity. eNeuro, 6(4). ENEURO.0498-18.2019. https://doi.org /10.1523/ENEURO.0498-18.2019

Bollimunta, A., Mo, J., Schroeder, C. E., \& Ding, M. (2011). Neuronal mechanisms and attentional modulation of corticothalamic alpha oscillations. The Journal of Neuroscience, 31(13), 4935-4943. https://doi.org/10.1523 /JNEUROSCI.5580-10.2011

Buonomano, D. V., \& Maass, W. (2009). State-dependent computations: Spatiotemporal processing in cortical networks. Nature Reviews Neuroscience, 10, 113-125. https://doi.org /10.1038/nrn2558

Byeon, J., Choi, T. Y., Won, G. H., Lee, J., \& Kim, J. W. (2020). A novel quantitative electroencephalography subtype with high alpha power in ADHD: ADHD or misdiagnosed ADHD? PLOS ONE, 15(11), e0242566. https://doi.org/10.1371 /journal.pone.0242566

Cannon, R. L. (2012). LORETA neurofeedback: Odd reports, observations, and findings associated with spatial specific neurofeedback training. Journal of Neurotherapy, 16(2), 164167. https://doi.org/10.1080/10874208.2012.677611

Cannon, R. L., Baldwin, D. R., Diloreto, D. J., Phillips, S. T., Shaw, T. L., \& Levy, J. J. (2014). LORETA neurofeedback in the precuneus: Operant conditioning in basic mechanisms of self-regulation. Clinical EEG and Neuroscience, 45(4), 238248. https://doi.org/10.1177\%2F1550059413512796

Cannon, R. L., Strunk, W., Carroll, S., \& Carroll, S. (2018). LORETA neurofeedback at precuneus in 3-year-old female with intrauterine drug exposure. NeuroRegulation, 5(2), 7582. https://doi.org/10.15540/nr.5.2.75
Capotosto, P., Spadone, S., Tosoni, A., Sestieri, C., Romani, G. L., Della Penna, S., \& Corbetta, M. (2015). Dynamics of EEG rhythms support distinct visual selection mechanisms in parietal cortex: A simultaneous transcranial magnetic stimulation and EEG study. The Journal of Neuroscience, 35(2), 721-730. https://doi.org/10.1523/jneurosci.206614.2015

Cassady, K., You, A., Doud, A., \& He, B. (2014). The impact of mind-body awareness training on the early learning of a brain-computer interface. Technology, 2(3), 254-260. https://doi.org/10.1142/S233954781450023X

Cavanna, A. E., \& Trimble, M. R. (2006). The precuneus: A review of its functional anatomy and behavioural correlates. Brain, 129(3), 564-583. https://doi.org/10.1093/brain/awl004

Chafee, M. V., \& Crowe, D. A. (2017). Implicit and explicit learning mechanisms meet in monkey prefrontal cortex. Neuron, 96(2), 256-258. https://doi.org/10.1016/j.neuron.2017.09.049

Cheng, M.-Y., Huang, C.-J., Chang, Y.-K., Koester, D., Schack, T., \& Hung, T.-M. (2015). Sensorimotor rhythm neurofeedback enhances golf putting performance. Journal of Sport and Exercise Psychology, 37(6), 626-636. https://doi.org/10.1123/jsep.2015-0166

Chiew, M., LaConte, S. M., \& Graham, S. J. (2012). Investigation of fMRI neurofeedback of differential primary motor cortex activity using kinesthetic motor imagery. Neurolmage, 61(1), 21-31. https://doi.org/10.1016/j.neuroimage.2012.02.053

Chow, T., Javan, T., Ros, T., \& Frewen, P. (2017). EEG dynamics of mindfulness meditation versus alpha neurofeedback: $A$ sham-controlled study. Mindfulness, 8, 572-584. https://doi.org/10.1007/S12671-016-0631-8

Collura, F. T., \& Siever, D. (2009). Audio-visual entrainment in relation to mental health and EEG. In T. H. Budzynski, H. K. Budzynski, J. R. Evans, \& A. Abarbanel (Eds.), Introduction to quantitative EEG and neurofeedback: Advanced theory and applications (2nd ed., pp. 195-220). Cambridge, MA: Academic Press.

Cooper, N. R., Croft, R. J., Dominey, S. J. J., Burgess, A. P., \& Gruzelier, J. H. (2003). Paradox lost? Exploring the role of alpha oscillations during externally vs. internally directed attention and the implications for idling and inhibition hypotheses. International Journal of Psychophysiology, 47(1), 65-74. https://doi.org/10.1016/s0167-8760(02)00107-1

Da Costa, N. M. C., Bicho, E. G., \& Dias, N. S. (2019). Does priming subjects, with not only resting state but also with mindfulness or/and guided imagery, affect self-regulation of SMR neurofeedback? Framework to improve brain selfregulation and support the rehabilitation of disorders such as depression, anxiety, stress and attention control. Frontiers in Cellular Neuroscience, Conference Abstract: XVI Meeting of the Portuguese Society for Neuroscience (SPN2019). https://doi.org/10.3389/conf.fncel.2019.01.00050

Da Costa, N. M. C., Bicho, E. G., \& Dias, N. S. (2020, August). Priming with mindfulness affects our capacity to self-regulate brain activity? Presented at the 2020 IEEE 8th International Conference on Serious Games and Applications for Health (SeGAH), Vancouver, BC, Canada, pp. 1-8. https://doi.org /10.1109/SeGAH49190.2020.9201841

Deiber, M.-P., Hasler, R., Colin, J., Dayer, A., Aubry, J.-M., Baggio, S., ... Ros, T. (2020). Linking alpha oscillations, attention and inhibitory control in adult ADHD with EEG neurofeedback. Neurolmage: Clinical, 25, 102145. https://doi.org/10.1016/j.nicl.2019.102145

Destrebecqz, A., Peigneux, P., Laureys, S., Degueldre, C., Del Fiore, G., Aerts, J., ... Maquet, P. (2005). The neural correlates of implicit and explicit sequence learning: Interacting networks revealed by the process dissociation procedure. Learning \& Memory, 12(5), 480-490. https://doi.org/10.1101//m.95605 
Doppelmayr, M., Klimesch, W., Stadler, W., Pöllhuber, D., \& Heine, C. (2002). EEG alpha power and intelligence. Intelligence, 30(3), 289-302. https://doi.org/10.1016/S01602896(01)00101-5

Doppelmayr, M., Klimesch, W., Sauseng, P., Hödlmoser, K., Stadler, W., \& Hanslmayr, S. (2005). Intelligence related differences in EEG-bandpower. Neuroscience Letters, 381(3), 309-313. https://doi.org/10.1016/j.neulet.2005.02.037

Eichenbaum, H. (1999). Conscious awareness, memory and the hippocampus. Nature Neuroscience, 2, 775-776. https://doi.org/10.1038/12137

Emmert, K., Kopel, R., Sulzer, J., Brühl, A. B., Berman, B. D., Linden, D. E. J., ... Haller, S. (2016). Meta-analysis of realtime fMRI neurofeedback studies using individual participant data: How is brain regulation mediated? Neurolmage, 124(A), 806-812. https://doi.org/10.1016/j.neuroimage.2015.09.042

Ergenoglu, T., Demiralp, T., Bayraktaroglu, Z., Ergen, M., Beydagi, H., \& Uresin, Y. (2004). Alpha rhythm of the EEG modulates visual detection performance in humans. Cognitive Brain Research, 20(3), 376-383. https://doi.org/10.1016 /j.cogbrainres.2004.03.009

Escolano, C., Aguilar, M., \& Minguez, J. (2011, August). EEGbased upper alpha neurofeedback training improves working memory performance. Presented at the 2011 Annual International Conference of the IEEE Engineering in Medicine and Biology Society, Boston, MA, pp. 2327-2330.

Faymonville, M.-E., Boly, M., \& Laureys, S. (2006). Functional neuroanatomy of the hypnotic state. Journal of PhysiologyParis, 99(4-6), 463-469. https://doi.org/10.1016 /j.jphysparis.2006.03.018

Fehmi, L. G., \& Robbins, J. (2008). The open-focus brain: harnessing the power of attention to heal mind and body. Boulder, CO: Shambhala Publications.

Fell, J., Elfadil, H., Klaver, P., Roschke, J., Elger, C. E., \& Fernández, G. (2002). Covariation of spectral and nonlinear EEG measures with alpha biofeedback. The International Journal of Neuroscience, 112(9), 1047-1057. https://doi.org $/ 10.1080 / 00207450290026049$

Fetz, E. E. (1969). Operant conditioning of cortical unit activity. Science, 163(3870), 955-958. Retrieved from https://depts.washington.edu/fetzweb/assets/fetz-science1969.pdf

Fink, A., Grabner, R. H., Benedek, M., \& Neubauer, A. C. (2006). Divergent thinking training is related to frontal electroencephalogram alpha synchronization. European Journal of Neuroscience, 23(8), 2241-2246. https://doi.org /10.1111/j.1460-9568.2006.04751.x

Fink, A., Grabner, R. H., Benedek, M., Reishofer, G., Hauswirth, V., Fally, M., ... Neubauer, A. C. (2009). The creative brain: Investigation of brain activity during creative problem solving by means of EEG and FMRI. Human Brain Mapping, 30(3), 734-748. https://doi.org/10.1002/hbm.20538

Foster, D. S. (1990). EEG and subjective correlates of alphafrequency binaural-beat stimulation combined with alpha biofeedback. Memphis State University. Retrieved from http://stealthskater.com/Documents/Lucid_07.pdf

Foxe, J. J., Simpson, G. V., \& Ahlfors, S. P. (1998). Parietooccipital $\sim 10 \mathrm{~Hz}$ activity reflects anticipatory state of visual attention mechanisms. NeuroReport, 9(17), 3929-3933. https://doi.org/10.1097/00001756-199812010-00030

Freedman, D. J., \& Ibos, G. (2018). An integrative framework for sensory, motor, and cognitive functions of the posterior parietal cortex. Neuron, 97(6), 1219-1234. https://doi.org /10.1016/j.neuron.2018.01.044

Gong, A., Nan, W., Yin, E., Jiang, C., \& Fu, Y. (2020). Efficacy, trainability, and neuroplasticity of SMR vs. alpha rhythm shooting performance neurofeedback training. Frontiers in Human Neuroscience, 14, 94. https://doi.org/10.3389 /fnhum.2020.00094
Graf, P., Squire, L. R., \& Mandler, G. (1984). The information that amnesic patients do not forget. Journal of Experimental Psychology: Learning, Memory, and Cognition, 10(1), 164178. https://doi.org/10.1037/0278-7393.10.1.164

Grahn, J. A., Parkinson, J. A., \& Owen, A. M. (2008). The cognitive functions of the caudate nucleus. Progress in Neurobiology, $\quad 86(3), \quad 141-155 . \quad$ https://doi.org/10.1016 /j.pneurobio.2008.09.004

Green, E., \& Green, A. (1977). Beyond biofeedback. New York, NY: Delacorte Press.

Gruzelier, J. H., \& Egner, T. (2004). Physiological self-regulation: Biofeedback and neurofeedback. In A. Williamon (Ed.), Musical excellence: Strategies and techniques to enhance performance (pp. 197-219). London, UK: Oxford University Press. /9780198525356.003.0011

Gulbinaite, R., van Viegen, T., Wieling, M., Cohen, M. X., \& VanRullen, R. (2017). Individual alpha peak frequency predicts $10 \mathrm{~Hz}$ flicker effects on selective attention. The Journal of Neuroscience, 37(42), 10173-10184. https://doi.org/10.1523/JNEUROSCI.1163-17.2017

Haegens, S., Händel, B. F., \& Jensen, O. (2011). Top-down controlled alpha band activity in somatosensory areas determines behavioral performance in a discrimination task. The Journal of Neuroscience, 31(14), 5197-5204. https://doi.org/10.1523/JNEUROSCI.5199-10.2011

Haegens, S., Osipova, D., Oostenveld, R., \& Jensen, O. (2010). Somatosensory working memory performance in humans depends on both engagement and disengagement of regions in a distributed network. Human Brain Mapping, 31(1), 26-35. https://doi.org/10.1002/hbm.20842

Haller, S., Birbaumer, N., \& Veit, R. (2010). Real-time fMRI feedback training may improve chronic tinnitus. European Radiology, 20, 696-703. https://doi.org/10.1007/s00330-0091595-z

Hammond, D. C. (2002). Clinical corner: The alpha rhythm in EEG. Journal of Neurotherapy, 6(3), 89-100. https://doi.org /10.1300/J184v06n03_09

Hammond, D. C. (2005). Neurofeedback treatment of depression and anxiety. Journal of Adult Development, 12, 131-137. https://doi.org/10.1007/s10804-005-7029-5

Hammond, D. C. (2011). What is neurofeedback: An update. Journal of Neurotherapy, 15(4), 305-336. https://doi.org /10.1080/10874208.2011.623090

Hammond, D. C., Stockdale, S., Hoffman, D., Ayers, M. E., \& Nash, J. (2001). Adverse reactions and potential iatrogenic effects in neurofeedback training. Journal of Neurotherapy, 4(4), 57-69. https://doi.org/10.1300/J184v04n04_09

HansImayr, S., Gross, J., Klimesch, W., \& Shapiro, K. L. (2011). The role of a oscillations in temporal attention. Brain Research Reviews, 67(1-2), 331-343. https://doi.org/10.1016 /j.brainresrev.2011.04.002

Hanslmayr, S., Sauseng, P., Doppelmayr, M., Schabus, M., \& Klimesch, W. (2005). Increasing individual upper alpha power by neurofeedback improves cognitive performance in human subjects. Applied Psychophysiology and Biofeedback, 30(1), 1-10. https://doi.org/10.1007/s10484-005-2169-8

Hardman, E., Gruzelier, J., Cheesman, K., Jones, C., Liddiard, D., Schleichert, H., \& Birbaumer, N. (1997). Frontal interhemispheric asymmetry: Self regulation and individual differences in humans. Neuroscience Letters, 221(2-3), 117120. https://doi.org/10.1016/s0304-3940(96)13303-6

Hardt, J. V., \& Gale, R. (1993, March). Creativity increases in scientists through alpha EEG feedback training. Proceedings of the Association for Applied Psychophysiology and Biofeedback, 24th Annual Meeting, Los Angeles, CA. Retrieved from https://cdn2.hubspot.net/hubfs/2414356 /Biocybernaut_Institute_Aug_2016/PDF/ScienceArticle_Creln cSci.pdf?t=1472687102807 
Hardt J. V., \& Kamiya J. (1978). Anxiety change through electroencephalographic alpha feedback seen only in high anxiety subjects. Science, 201(4350), 79-81. https://doi.org $/ 10.1126 /$ science.663641

Haxby, J. V., Horwitz, B., Ungerleider, L. G., Maisog, J. M., Pietrini, P., \& Grady, C. L. (1994). The functional organization of human extrastriate cortex: A PET-rCBF study of selective attention to faces and locations. The Journal of Neuroscience, 14(11), 6336-6353. https://doi.org/10.1523/JNEUROSCI.1411-06336.1994

Heindel, W. C., Salmon, D. P., Shults, C. W., Walicke, P. A., \& Butters, N. (1989). Neuropsychological evidence for multiple implicit memory systems: A comparison of Alzheimer's, Huntington's, and Parkinson's disease patients. The Journal of Neuroscience, 9(2), 582-587. https://doi.org/10.1523 /JNEUROSCI.09-02-00582.1989

Ibric, V. L., \& Davis, C. J. (2007). The ROSHI in neurofeedback. In J. R. Evans (Ed.), Handbook of neurofeedback: Dynamics and clinical applications (pp. 185-211). New York, NY: Haworth Medical Press.

Jarbo, K., \& Verstynen, T. D. (2015). Converging structural and functional connectivity of orbitofrontal, dorsolateral prefrontal, and posterior parietal cortex in the human striatum. The Journal of Neuroscience, 35(9), 3865-3878. https://doi.org /10.1523/JNEUROSCI.2636-14.2015

Jaušovec, N. (1996). Differences in EEG alpha activity related to giftedness. Intelligence, 23(3), 159-173. https://doi.org /10.1016/S0160-2896(96)90001-X

Jelic, V., Johansson, S.-E., Almkvist, O., Shigeta, M., Julin, P., Nordberg, A., ... Wahlund, L.-O. (2000). Quantitative electroencephalography in mild cognitive impairment: Longitudinal changes and possible prediction of Alzheimer's disease. Neurobiology of Aging, 21(4), 533-540. https://doi.org/10.1016/s0197-4580(00)00153-6

Jensen, O., \& Mazaheri, A. (2010). Shaping functional architecture by oscillatory alpha activity: Gating by inhibition. Frontiers in Human Neuroscience, 4, 186. https://doi.org /10.3389/fnhum.2010.00186

Kawashima, R., O'Sullivan, B. T., \& Roland, P. E. (1995). Positron-emission tomography studies of cross-modality inhibition in selective attentional tasks: Closing the "mind's eye." Proceedings of the National Academy of Sciences of the United States of America, 92(13), 5969-5972. https://doi.org/10.1073/pnas.92.13.5969

Kelly, S. P., Lalor, E. C., Reilly, R. B., \& Foxe, J. J. (2006). Increases in alpha oscillatory power reflect an active retinotopic mechanism for distracter suppression during sustained visuospatial attention. Journal of Neurophysiology, 95(6), 3844-3851. https://doi.org/10.1152/jn.01234.2005

Kerson, C., Sherman, R. A., \& Kozlowski, G. P. (2009). Alpha suppression and symmetry training for generalized anxiety symptoms. Journal of Neurotherapy, 13(3), 146-155. https://doi.org/10.1080/10874200903107405

Kirsch, D. L., \& Nichols, F. (2013). Cranial electrotherapy stimulation for treatment of anxiety, depression, and insomnia. Psychiatric Clinics of North America, 36(1), 169176. https://doi.org/10.1016/j.psc.2013.01.006

Klimesch, W. (1999). EEG alpha and theta oscillations reflect cognitive and memory performance: A review and analysis. Brain Research Reviews, 29(2-3), 169-195. https://doi.org/10.1016/S0165-0173(98)00056-3

Klimesch, W., Doppelmayr, M., \& HansImayr, S. (2006). Upper alpha ERD and absolute power: Their meaning for memory performance. Progress in Brain Research, 159, 151-165. https://doi.org/10.1016/S0079-6123(06)59010-7

Klimesch, W., Doppelmayr, M., Pachinger, T., \& Ripper, B. (1997). Brain oscillations and human memory: EEG correlates in the upper alpha and theta band. Neuroscience
Letters, 238(1-2), 9-12. https://doi.org/10.1016/s03043940(97)00771-4

Klimesch, W., Doppelmayr, M., Röhm, D., Pöllhuber, D., \& Stadler, W. (2000). Simultaneous desynchronization and synchronization of different alpha responses in the human electroencephalograph: A neglected paradox? Neuroscience Letters, 284(1-2), 97-100. https://doi.org/10.1016/S03043940(00)00985-X

Klimesch, W., Doppelmayr, M., Russegger, H., Pachinger, T., \& Schwaiger, J. (1998). Induced alpha band power changes in the human EEG and attention. Neuroscience Letters, 244(2), 73-76. https://doi.org/10.1016/S0304-3940(98)00122-0

Klimesch, W., Sauseng, P., \& Hanslmayr, S. (2007). EEG alpha oscillations: The inhibition-timing hypothesis. Brain Research Reviews, 53(1), 63-88. https://doi.org/10.1016 /j.brainresrev.2006.06.003

Klimesch, W., Schack, B., Schabus, M., Doppelmayr, M., Gruber, W., \& Sauseng, P. (2004). Phase-locked alpha and theta oscillations generate the $\mathrm{P} 1-\mathrm{N} 1$ complex and are related to memory performance. Cognitive Brain Research, 19(3), 302316. https://doi.org/10.1016/j.cogbrainres.2003.11.016

Knowlton, B. J., Mangels, J. A., \& Squire, L. R. (1996). A neostriatal habit learning system in humans. Science, 273(5280), 1399-1402. https://doi.org/10.1126 /science.273.5280.1399

Knyazev, G. G., Savostyanov, A. N., \& Levin, E. A. (2006). Alpha synchronization and anxiety: Implications for inhibition vs. alertness hypotheses. International Journal of Psychophysiology, 59(2), 151-158. https://doi.org/10.1016 /j.jpsycho.2005.03.025

Kobayashi, S., Schultz, W., \& Sakagami, M. (2010). Operant conditioning of primate prefrontal neurons. Journal of Neurophysiology, 103(4), 1843-1855. https://doi.org/10.1152 /jn.00173.2009

Kober, S. E., Schweiger, D., Witte, M., Reichert, J. L., Grieshofer, P., Neuper, C., \& Wood, G. (2015). Specific effects of EEG based neurofeedback training on memory functions in poststroke victims. Journal of NeuroEngineering and Rehabilitation, 12, 107. https://doi.org/10.1186/s12984-0150105-6

Kober, S. E., Witte, M., Ninaus, M., Koschutnig, K., Wiesen, D., Zaiser, G., ... Wood, G. M. (2017). Ability to gain control over one's own brain activity and its relation to spiritual practice: A multimodal imaging study. Frontiers in Human Neuroscience, 11, 271. https://doi.org/10.3389/fnhum.2017.00271

Kober, S. E., Witte, M., Ninaus, M., Neuper, C., \& Wood, G. (2013). Learning to modulate one's own brain activity: The effect of spontaneous mental strategies. Frontiers in Human Neuroscience, $7, \quad 695.6$ https://doi.org/10.3389 /fnhum.2013.00695

Konen, C. S., \& Haggard, P. (2014). Multisensory parietal cortex contributes to visual enhancement of touch in humans: A single-pulse TMS study. Cerebral Cortex, 24(2), 501-507. https://doi.org/10.1093/cercor/bhs331

Koralek, A. C., Jin, X., Long, J. D., II, Costa, R. M., \& Carmena, J. M. (2012). Corticostriatal plasticity is necessary for learning intentional neuroprosthetic skills. Nature, 483(7389), 331335. https://doi.org/10.1038/nature 10845

Kropotov, J. D. (2009). Quantitative EEG, event-related potentials and neurotherapy. Cambridge, MA: Academic Press.

Lam, S.-L., Criaud, M., Alegria, A., Barker, G. J., Giampietro, V., \& Rubia, K. (2020). Neurofunctional and behavioural measures associated with fMRI-neurofeedback learning in adolescents with attention-deficit/hyperactivity disorder. Neurolmage: Clinical, 27, 102291. https://doi.org/10.1016 /j.nicl.2020.102291

Lawrence, E. J., Su, L., Barker, G. J., Medford, N., Dalton, J., Williams, S. C. R., ... David, A. S. (2014). Self-regulation of 
the anterior insula: Reinforcement learning using real-time fMRI neurofeedback. Neurolmage, 88, 113-124. https://doi.org/10.1016/j.neuroimage.2013.10.069

Liljeholm, M., \& O'Doherty, J. P. (2012). Contributions of the striatum to learning, motivation, and performance: An associative account. Trends in Cognitive Sciences, 16(9), 467-475. https://doi.org/10.1016/j.tics.2012.07.007

London, P., Hart, J. T., \& Leibovitz, M. P. (1968). EEG alpha rhythms and susceptibility to hypnosis. Nature, 219(5149), 71-72. https://doi.org/10.1038/219071a0

Lustenberger, C., Boyle, M. R., Foulser, A. A., Mellin, J. M., \& Fröhlich, F. (2015). Functional role of frontal alpha oscillations in creativity. Cortex, 67, 74-82. https://doi.org/10.1016 /j.cortex.2015.03.012

McNorgan, C. (2012). A meta-analytic review of multisensory imagery identifies the neural correlates of modality-specific and modality-general imagery. Frontiers in Human Neuroscience, $\quad 6, \quad 285 . \quad$ https://doi.org/10.3389 /fnhum.2012.00285

Meeuwissen, E. B., Takashima, A., Fernández, G., \& Jensen, O. (2011). Increase in posterior alpha activity during rehearsal predicts successful long-term memory formation of word sequences. Human Brain Mapping, 32(12), 2045-2053. https://doi.org/10.1002/hbm.21167

Milner, B., Corkin, S., \& Teuber, H.-L. (1968). Further analysis of the hippocampal amnesic syndrome: 14-year follow-up study of H.M. Neuropsychologia, 6(3), 215-234. https://doi.org /10.1016/0028-3932(68)90021-3

Mohagheghi, A., Amiri, S., Bonab, N. M., Chalabianloo, G., Noorazar, S. G., Tabatabaei, S. M., \& Farhang, S. (2017). A randomized trial of comparing the efficacy of two neurofeedback protocols for treatment of clinical and cognitive symptoms of ADHD: Theta suppression/beta enhancement and theta suppression/alpha enhancement. BioMed Research International, 2017, 3513281. https://doi.org/10.1155/2017/3513281

Moriyama, T. S., Polanczyk, G., Caye, A., Banaschewski, T., Brandeis, D., \& Rohde, L. A. (2012). Evidence-based information on the clinical use of neurofeedback for ADHD. Neurotherapeutics, 9, 588-598. https://doi.org/10.1007 /s13311-012-0136-7

Nan, W., Rodrigues, J. P., Ma, J., Qu, X., Wan, F., Mak, P.-I., ... Rosa, A. (2012). Individual alpha neurofeedback training effect on short term memory. International Journal of Psychophysiology, 86(1), 83-87. https://doi.org/10.1016 j.ijpsycho.2012.07.182

Niedermeyer, E. (1999). The normal EEG of the waking adult. In E. Niedermeyer \& F. Lopes Da Silva (Eds.), Electroencephalography: Basic principles, clinical applications, and related fields (pp. 149-173). Baltimore, MD: Lippincott, Williams \& Wilkins. Retrieved from http://www.ccs.fau.edu/ bressler/EDU/NTSA/References/Nie dermeyer_1999.pdf

Ninaus, M., Kober, S. E., Witte, M., Koschutnig, K., Stangl, M., Neuper, C., \& Wood, G. (2013). Neural substrates of cognitive control under the belief of getting neurofeedback training. Frontiers in Human Neuroscience, 7, 914. https://doi.org/10.3389/fnhum.2013.00914

Niv, S. (2013). Clinical efficacy and potential mechanisms of neurofeedback. Personality and Individual Differences, 54(6), 676-686. https://doi.org/10.1016/j.paid.2012.11.037

Norris, S. L., Lee, C.-T., Burshteyn, D., \& Cea-Aravena, J. (2000). The effects of performance enhancement training on hypertension, human attention, stress, and brain wave patterns. Journal of Neurotherapy, 4(3), 29-44. https://doi.org /10.1300/J184v04n03 03

Norris, S. L., Lee, C.-T., Cea, J., \& Burshteyn, D. (1998). Performance enhancement training effects on attention.
Journal of Neurotherapy, 3(1), 19-25. https://doi.org/10.1300 IJ184v03n01_03

Packard, M. G., \& Knowlton, B. J. (2002). Learning and memory functions of the basal ganglia. Annual Review of Neuroscience, 25, 563-593. https://doi.org/10.1146 lannurev.neuro.25.112701.142937

Peigneux, P., Laureys, S., Delbeuck, X., \& Maquet, P. (2001). Sleeping brain, learning brain. The role of sleep for memory systems. NeuroReport, 12(18), A111-A124. https://doi.org $/ 10.1097 / 00001756-200112210-00001$

Peniston, E. G., \& Kulkosky, P. J. (1991). Alpha-theta brainwave neurofeedback for Vietnam veterans with combat-related post-traumatic stress disorder. Medical Psychotherapy, 4, 4760. Retrieved from https://giuliotarantino.com/wp-content /uploads/2020/04/penistonptsd-peniston1989.pdf

Peters, S., \& Crone, E. A. (2017). Increased striatal activity in adolescence benefits learning. Nature Communications, 8, 1983. https://doi.org/10.1038/s41467-017-02174-z

Pfurtscheller, G., Stancák, A., \& Neuper, C. (1996). Event-related synchronization (ERS) in the alpha band-an electrophysiological correlate of cortical idling: A review. International Journal of Psychophysiology, 24(1-2), 39-46. https://doi.org/10.1016/S0167-8760(96)00066-9

Poldrack, R. A., Clark, J., Paré-Blagoev, E. J., Shohamy, D., Moyano, J. C., Myers, C., \& Gluck, M. A. (2001). Interactive memory systems in the human brain. Nature, 414, 546-550. https://doi.org/10.1038/35107080

Putman, J. A. (2000). The effects of brief, eyes-open alpha brain wave training with audio and video relaxation induction on the EEG of 77 Army reservists. Journal of Neurotherapy, 4(1), 17-28. https://doi.org/10.1300/J184v04n01_03

Raichle, M. E., MacLeod, A. M., Snyder, A. Z.., Powers, W. J., Gusnard, D. A., \& Shulman, G. L. (2001). A default mode of brain function. Proceedings of the National Academy of Sciences of the United States of America, 98(2), 676-682. https://doi.org/10.1073/pnas.98.2.676

Ramot, M., Grossman, S., Friedman, D., \& Malach, R. (2016). Covert neurofeedback without awareness shapes cortical network spontaneous connectivity. Proceedings of the National Academy of Sciences of the United States of America, 113(17), E2413-E2420. https://doi.org/10.1073 /pnas. 1516857113

Rauch, S. L., Whalen, P. J., Savage, C. R., Curran, T., Kendrick, A., Brown, H. D., ... Rosen, B. R. (1997). Striatal recruitment during an implicit sequence learning task as measured by functional magnetic resonance imaging. Human Brain Mapping, 5(2), 124-132. https://doi.org/10.1002/(SICl)10970193(1997)5:2<124::AID-HBM6>3.0.CO;2-5

Ray, W. J., \& Cole, H. W. (1985). EEG alpha activity reflects attentional demands, and beta activity reflects emotional and cognitive processes. Science, 228(4700), 750-752. https://doi.org/10.1126/science.3992243

Reber, P. J., \& Squire, L. R. (1994). Parallel brain systems for learning with and without awareness. Learning \& Memory, 1(4), 217-229. Retrieved from http://learnmem.cshlp.org /content/1/4/217.full.pdf

Rees, G., Frith, C. D., \& Lavie, N. (1997). Modulating irrelevant motion perception by varying attentional load in an unrelated task. Science, 278(5343), 1616-1619. https://doi.org/10.1126 /science.278.5343.1616

Rohrmeier, M., \& Rebuschat, P. (2012). Implicit learning and acquisition of music. Topics in Cognitive Science, 4(4), 525553. https://doi.org/10.1111/j.1756-8765.2012.01223.x

Romei, V., Gross, J., \& Thut, G. (2010). On the role of prestimulus alpha rhythms over occipito-parietal areas in visual input regulation: Correlation or causation? The Journal of Neuroscience, 30(25), 8692-8697. https://doi.org/10.1523 /jneurosci.0160-10.2010 
Ros, T., Michela, A., Bellman, A., Vuadens, P., Saj, A., \& Vuilleumier, P. (2017). Increased alpha-rhythm dynamic range promotes recovery from visuospatial neglect: A neurofeedback study. Neural Plasticity, 2017, 7407241. https://doi.org/10.1155/2017/7407241

Rugg, M. D., Mark, R. E., Walla, P., Schloerscheidt, A. M., Birch, C. S., \& Allan, K. (1998). Dissociation of the neural correlates of implicit and explicit memory. Nature, 392(6676), 595-598. https://doi.org/10.1038/33396

Sauseng, P., Klimesch, W., Heise, K. F., Gruber, W. R., Holz, E., Karim, A. A., ... Hummel, F. C. (2009). Brain oscillatory substrates of visual short-term memory capacity. Current Biology, 19(21), 1846-1852. https://doi.org/10.1016 /j.cub.2009.08.062

Saxby, E., \& Peniston, E. G. (1995). Alpha-theta brainwave neurofeedback training: An effective treatment for male and female alcoholics with depressive symptoms. Journal of Clinical Psychology, 51(5), 685-693. https://doi.org/10.1002 /1097-4679(199509)51:5<685::AIDJCLP2270510514>3.0.CO;2-K

Scharnowski, F., Veit, R., Zopf, R., Studer, P., Bock, S., Diedrichsen, J., ... Weiskopf, N. (2015). Manipulating motor performance and memory through real-time fMRI neurofeedback. Biological Psychology, 108, 85-97. https://doi.org/10.1016/j.biopsycho.2015.03.009

Seger, C. A., Prabhakaran, V., Poldrack, R. A., \& Gabrieli, J. D. E. (2000). Neural activity differs between explicit and implicit learning of artificial grammar strings: An fMRI study. Psychobiology, 28(3), 283-292. https://doi.org/10.3758 /BF03331987

Sherlin, L. (2009). Diagnosing and treating brain function through the use of low resolution brain electromagnetic tomography (LORETA). In T. H. Budzynski, H. K. Budzynski, J. R. Evans, \& A. Abarbanel (Eds.), Introduction to quantitative EEG and neurofeedback: Advanced theory and applications (2nd ed., pp. 83-101). Cambridge, MA: Academic Press.

Sherlin, L. H., Arns, M., Lubar, J., Heinrich, H., Kerson, C., Strehl, U., \& Sterman, M. B. (2011). Neurofeedback and basic learning theory: Implications for research and practice. Journal of Neurotherapy, 15(4), 292-304. https://doi.org $/ 10.1080 / 10874208.2011 .623089$

Sigala, R., Haufe, S., Roy, D., Dinse, H. R., \& Ritter, P. (2014). The role of alpha-rhythm states in perceptual learning: Insights from experiments and computational models. Frontiers in Computational Neuroscience, 8, 36. https://doi.org/10.3389/fncom.2014.00036

Sitaram, R., Ros, T., Stoeckel, L., Haller, S., Scharnowski, F., Lewis-Peacock, J., ... Sulzer, J. (2017). Closed-loop brain training: The science of neurofeedback. Nature Reviews Neuroscience, $\quad 18(2), \quad 86-100 . \quad$ https://doi.org/10.1038 /nrn.2016.164

Squire, L. R., \& Zola, S. M. (1996). Structure and function of declarative and nondeclarative memory systems. Proceedings of the National Academy of Sciences of the United States of America, 93(24), 13515-13522. https://doi.org/10.1073/pnas.93.24.13515

Sterman, M. B. (1977). Sensorimotor EEG operant conditioning: Experimental and clinical effects. Pavlovian Journal of Biological Science, 12(2), 63-92. https://doi.org/10.1007 /BF03004496

Sterman, M. B., \& Egner, T. (2006). Foundation and practice of neurofeedback for the treatment of epilepsy. Applied Psychophysiology and Biofeedback, 31(1), 21-35. https://doi.org/10.1007/s10484-006-9002-x

Stieger, J. R., Engel, S., Jiang, H., Cline, C. C., Kreitzer, M. J., \& $\mathrm{He}$, B. (2021). Mindfulness improves brain-computer interface performance by increasing control over neural activity in the alpha band. Cerebral Cortex, 31(1), 426-438. https://doi.org/10.1093/cercor/bhaa234
Sulzer, J., Sitaram, R., Blefari, M. L., Kollias, S., Birbaumer, N., Stephan, K. E., ... Gassert, R. (2013). Neurofeedbackmediated self-regulation of the dopaminergic midbrain. Neurolmage, $\quad 83, \quad 817-825 . \quad$ https://doi.org/10.1016 /j.neuroimage.2013.05.115

Surwillo, W. W. (1961). Frequency of the 'alpha' rhythm, reaction time and age. Nature, 191, 823-824. https://doi.org/10.1038 $/ 191823 \mathrm{a} 0$

Thompson, M., \& Thompson, L. (2009). Asperger's syndrome intervention: Combining neurofeedback, biofeedback and metacognition. In T. H. Budzynski, H. K. Budzynski, J. R. Evans, \& A. Abarbanel (Eds.), Introduction to quantitative EEG and neurofeedback: Advanced theory and applications (2nd ed., pp. 365-410). Cambridge, MA: Academic Press.

Thut, G., Nietzel, A., Brandt, S. A., \& Pascual-Leone, A. (2006). $\alpha$-band electroencephalographic activity over occipital cortex indexes visuospatial attention bias and predicts visual target detection. The Journal of Neuroscience, 26(37), 9494-9502. https://doi.org/10.1523/JNEUROSCI.0875-06.2006

Travis, T. A., Kondo, C. Y., \& Knott, J. R. (1974). Alpha conditioning, A controlled study. Journal of Nervous and Mental Disease, 158(3), 163-173. https://doi.org/10.1097 /00005053-197403000-00001

Tulving, E. (1972). Episodic and semantic memory. In E. Tulving, \& W. Donaldson (Eds.), Organization of memory (pp. 381402). Cambridge, MA: Academic Press. Retrieved from http://alumni.media.mit.edu/ jorkin/generals/papers/Tulving_ memory.pdf

van Dijk, H., Schoffelen, J.-M., Oostenveld, R., \& Jensen, O. (2008). Prestimulus oscillatory activity in the alpha band predicts visual discrimination ability. The Journal of Neuroscience, 28(8), 1816-1823. https://doi.org/10.1523 /JNEUROSCI.1853-07.2008

Veit, R., Singh, V., Sitaram, R., Caria, A., Rauss, K., \& Birbaumer, N. (2012). Using real-time $\mathrm{fMRI}$ to learn voluntary regulation of the anterior insula in the presence of threat-related stimuli. Social Cognitive and Affective Neuroscience, 7(6), 623-634. https://doi.org/10.1093/scan/nsr061

Vernon, D., Dempster, T., Bazanova, O. M., Rutterford, N., Pasqualini, M., \& Andersen, S. (2009). Alpha neurofeedback training for performance enhancement: Reviewing the methodology. Journal of Neurotherapy, 13(4), 214-227. https://doi.org/10.1080/10874200903334397

Verstraeten, E., \& Cluydts, R. (2002). Attentional switchingrelated human EEG alpha oscillations. NeuroReport, 13(5), 681-684. https://doi.org/10.1097/00001756-20020416000029

von Stein, A., \& Sarnthein, J. (2000). Different frequencies for different scales of cortical integration: From local gamma to long range alpha/theta synchronization. International Journal of Psychophysiology, 38(3), 301-313. https://doi.org/10.1016 /s0167-8760(00)00172-0

Williams, J. D., \& Gruzelier, J. H. (2001). Differentiation of hypnosis and relaxation by analysis of narrow band theta and alpha frequencies. International Journal of Clinical and Experimental Hypnosis, 49(3), 185-206. https://doi.org $/ 10.1080 / 00207140108410070$

Witte, M., Kober, S. E., Ninaus, M., Neuper, C., \& Wood, G. (2013). Control beliefs can predict the ability to up-regulate sensorimotor rhythm during neurofeedback training. Frontiers in Human Neuroscience, 7, 478. https://doi.org/10.3389 /fnhum.2013.00478

Wokke, M. E., \& Ro, T. (2019). Competitive frontoparietal interactions mediate implicit inferences. The Journal of Neuroscience, 39(26), 5183-5194. https://doi.org/10.1523 /JNEUROSCI.2551-18.2019

Wood, G., Kober, S. E., Witte, M., \& Neuper, C. (2014). On the need to better specify the concept of "control" in braincomputer-interfaces/neurofeedback research. Frontiers in 
Systems Neuroscience, 8, 171. https://doi.org/10.3389 /fnsys.2014.00171

Worden, M. S., Foxe, J. J., Wang, N., \& Simpson, G. V. (2000). Anticipatory biasing of visuospatial attention indexed by retinotopically specific a-band electroencephalography increases over occipital cortex. The Journal of Neuroscience, 20(6), 1-6. https://doi.org/10.1523/JNEUROSCI.20-06j0002.2000

Wu, X., \& Liu, X. Q. (1995). Study of the alpha frequency band of healthy adults in quantitative EEG. Clinical Electroencephalography, 26(2), 131-136. https://doi.org $/ 10.1177 / 155005949502600212$

Yang, J., \& Li, P. (2012). Brain networks of explicit and implicit learning. PLOS ONE, 7(8), e42993. https://doi.org/10.1371 /journal.pone.0042993

Yin, H. H., \& Knowlton, B. J. (2006). The role of the basal ganglia in habit formation. Nature Reviews Neuroscience, 7, 464476. https://doi.org/10.1038/nrn1919
Zoefel, B., Huster, R. J., \& Herrmann, C. S. (2011). Neurofeedback training of the upper alpha frequency band in EEG improves cognitive performance. Neurolmage, 54(2), 1427-1431. https://doi.org/10.1016/j.neuroimage.2010.08.078

Zotev, V., Misaki, M., Phillips, R., Wong, C. K., \& Bodurka, J. (2018). Real-time fMRI neurofeedback of the mediodorsal and anterior thalamus enhances correlation between thalamic BOLD activity and alpha EEG rhythm. Human Brain Mapping, 39(2), 1024-1042. https://doi.org/10.1002/hbm.23902

Received: January 15, 2021

Accepted: January 28, 2021

Published: March 29, 2021 